\title{
Percepción sobre la práctica de la aplicación de sonda urinaria con simulador clínico
}

\author{
Perception about the practice of urinary catheter application with clinical simulator
} Clara Carlson Morales ${ }^{a}$, Mayra L. Sánchez Ruíz y Kerem D. Gómez Estrada ${ }^{c}$

\begin{abstract}
:
The purpose of this research was to know the perception regarding the willingness to use a low-cost prototype to perform clinical simulation practices specifically to learn the application of urinary catheters. Therefore, a descriptive exploratory survey was applied, from October 15 to 30, 2018. Among the main results, it was discovered that $41 \%$ of the respondent's state that they have seen the procedure of placement of the bladder catheter, but not performed. And 30\% say they have seen and performed such a procedure, so it was concluded that it is feasible to take action to carry out the didactic prototype so that more students are involved in learning. Currently considered as a trend in learning tools, simulators facilitate the understanding and acquisition of knowledge and skills. So, respondents became aware and were motivated to make and use prototypes as a teaching tool in clinical simulation.
\end{abstract}

\section{Keywords:}

Perception, Practices, Urinary Probe, Simulator

\section{Resumen:}

El propósito de esta investigación fue conocer la percepción respecto a la disposición de utilizar un prototipo de bajo costo para realizar prácticas en la simulación clínica de forma específica para aprender la aplicación de sonda urinaria. Por lo que se aplicó una encuesta de forma exploratoria descriptiva, del 15 al 30 de octubre del 2018. Entre los principales resultados se descubrió que el $41 \%$ de los encuestados manifiestan haber visto el procedimiento de colocación de sonda vesical, más no realizado. Y el 30\% expresan haber visto y realizo tal procedimiento, por lo que se concluyó que sí es viable tomar acción para realizar el prototipo didáctico para que más estudiantes se involucren en el aprendizaje. Considerada actualmente como de tendencia en las herramientas de aprendizajes, los simuladores facilitan la comprensión y adquisición de conocimientos y habilidades. Por lo que los encuestados hicieron conciencia y se motivaron para realizar y utilizar prototipos como herramienta didáctica en la simulación clínica.

\section{Palabras Clave:}

Percepción, Prácticas, Sonda Urinaria, Simulador.

\footnotetext{
${ }^{a}$ Autor de Correspondencia, Universidad Linda Vista, https://orcid.org/0000-0002-9752-8033, Email: clarita.carlson@ulv.edu.mx

b Universidad Linda Vista, https://orcid.org/0000-0001-8867-1025, Email: mayra.sanchez@ulv.edu.mx

b Universidad Linda Vista, https://orcid.org/0000-0001-5782-7760, Email: Kerem.gomez@ulv.edu.mx
} 


\section{Introducción}

La simulación clínica es una recreación en un escenario ideado para experimentar, esto ha favorecido el desarrollo acelerado de la simulación en la Educación Médica a nivel mundial (1). La simulación ha sido usada desde hace décadas en la enseñanza curricular de la enfermería (2), el profesional en salud debe ser una persona experimentada para realizar el procedimiento de sonda urinaria. En México son escasos las universidades e instituciones de salud que disponen de simuladores clínicos de sonda urinaria; y en el mercado cuando se encuentra son de difícil adquisición (3).

Por lo que la decisión de conocer la percepción de los estudiantes respecto al uso e importancia para ellos, de aprender y experimentar con simuladores, creemos que es de utilidad para la toma de decisión a corto plazo para incursionar y diseñar, esbozar, un simulador o prototipo que será amigable para realizar prácticas con este tipo de herramienta que facilitan el aprendizaje.

"El docente hace uso de diversos recursos didácticos entre ellos se encuentra la categoría de materiales de simulación, en la cual puede usar para la enseñanza aprendizaje maniquíes y simuladores de diversas técnicas". (4)

Consideramos que es necesario rescatar un concepto relacionado a la simulación en el ámbito educativo para cubrir los requisitos de experimentación o práctica clínica., creemos muy apropiado para este caso, el concepto expresado por los doctores Melchor Sánchez y Adrián Martínez, como la "recrear una situación clínica en un ambiente controlado, de seguridad psicológica, para el participante, sin entrar en contacto directo con el paciente con un objeto de aprendizaje o de evaluación". (5).

También conocido con el término modelos anatómicos resulta interesante que la Revolución convirtió los modelos anatómicos en valioso recurso didáctico para la enseñanza del arte de curar...Asimismo, los modelos anatómicos se volvieron los mediadores operacionales entre la realidad física del cuerpo y los procedimientos curativos de los futuros cirujanos, adquiriendo un gran valor en la comprensión y dominio de los procesos vitales (6).

El término prototipo, lo consideramos como lo define la Real Academia Española "Ejemplar original o primer molde en que se fabrica una figura u otra cosa."(7). también es interesante como lo define la enciclopedia cubana al decir que "es un enfoque de construir un poco y probar un poco antes de construir el Sistema final. Es un modelo a escala o facsímil de lo real, pero no tan funcional para que equivalga a un producto final, ya que no lleva a cabo la totalidad de las funciones necesarias del sistema final. (8).

Una de las normas relacionadas a este prototipo, de las cuales los estudiantes deben tener conocimientos previos Es la Norma Oficial Mexicana: NOM-052-SSA1-93. Que establece las especificaciones sanitarias de las sondas para drenaje urinario de hule látex natural estéril modelo Foley. (www.salud.gob.mx, s.f.)(9).

Algunos laboratorios de prácticas académicas de instituciones educativas, tienen entre su reglamento interno, la indicación que, en la medida de lo posible, los estudiantes no deben realizar prácticas en categoría de invasivas entre sí. Tal es el caso de la Universidad de Puebla (UniPuebla), plantel Sur, a quien me permito mencionar como ejemplo, ya que, en su reglamento de laboratorio de enfermería y práctica simulada, en la sección de normas relacionadas con la bioseguridad el punto 12, dice claramente "Queda estrictamente prohibido a los asesores realizar prácticas invasivas con los alumnos, ya que representa poner en riesgo su seguridad e integridad". (UdePuebla.mx, 2020) (10). Y eso, hace que los docentes y estudiantes busquen otras formas de realizar sus prácticas, siendo la simulación una buena opción.

\section{Objetivo}

Conocer la percepción sobre la creación de un prototipo básico que permita realizar la simulación de las prácticas de sonda urinaria, con la intención de facilitar a los estudiantes el aprendizaje para mejorar los conocimientos, las habilidades y así evitar o reducir los riesgos, como el de provocar alguna infección o en caso extremo y comprometer la vida del paciente.

\section{Metodología}

Para conocer la percepción de los estudiantes, maestros y personal de salud, se diseñó y aplicó una encuesta con 11 ítems, el estudio fue realizado de forma transversal, cuantitativa, descriptivo, cuasi experimental. Población conformada por 100 personas en el área de la salud entre las edades de 15 y 40 años, participando de la muestra algunos docentes y estudiantes de la Escuela de Enfermería, así como personal del consultorio médico escolar de la Universidad Linda Vista, a la par se aplicó a personal que labora en el Centro de Salud, de Pueblo Nuevo Solistahuacán, Chiapas. Los datos se recolectaron mediante una encuesta autoadministrada, con un tiempo promedio de respuesta de 4.73 minutos. Los datos se analizaron de forma tradicional con Excel. 
Para facilitar la réplica e incluso de mejora posterior de la obtención de información básica que se consultó se comparte el primer cuestionario, para ello ver la figura siguiente.

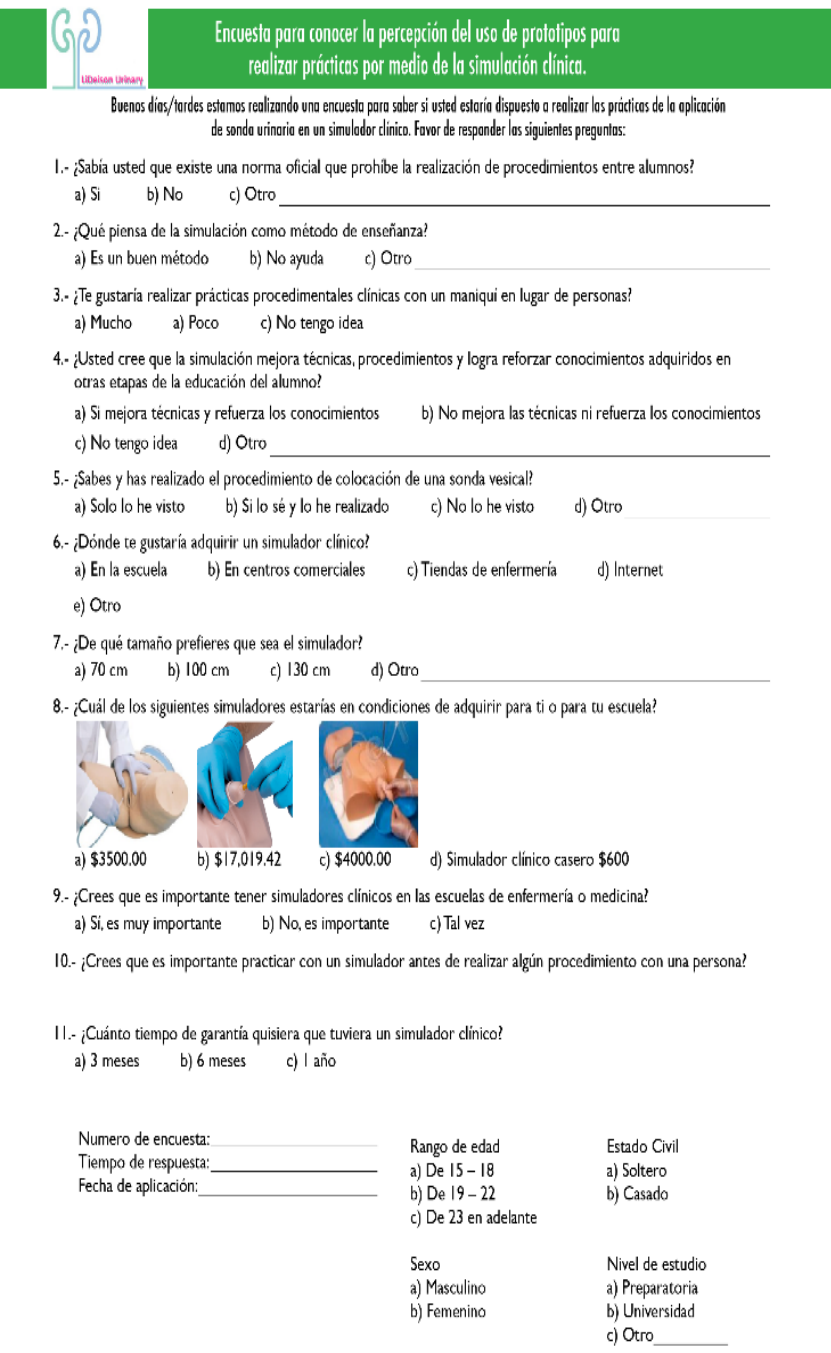

Figura 1. Elaboración propia. Las imágenes de la pregunta 8 fueron tomadas de: a) https://pranatec.com.mx/b) https://www.a3bs.com/

c) https://materialmedico24.es/l (11, 12 y 13$)$.

\section{Principales Resultados}

\section{Para entender mejor los principales resultados consideramos necesario compartir primero los generales, como se muestra en la siguiente figura.}

\begin{tabular}{|c|c|}
\hline Resultados & Porcentaje \\
\hline $\begin{array}{l}\text { 1.- ¿Sabía usted que existe una norma oficial que prohibe la realización de procedimientos } \\
\text { entre alumnos? }\end{array}$ & $51 \%$ \\
\hline 2.- ¿Qué piensa de la simulación como método de enseñanza? & $84 \%$ \\
\hline $\begin{array}{l}\text { 3.- Te gustaria realizar prácticas procedimentales clínicas con un maniquien lugar de } \\
\text { personas? }\end{array}$ & $49 \%$ \\
\hline $\begin{array}{l}\text { 4.- ¿Usted cree que la simulación mejora técnicas, procedimientos y logra reforzar } \\
\text { conocimientos adquiridos en otras etapas de la educación del alumno? }\end{array}$ & $85 \%$ \\
\hline 5.- ¿Sabes y has realizado el procedimiento de colocación de una sonda vesical? & $41 \%$ \\
\hline 6. ¿Dónde te gustaría adquirir un simulador clinico? & $44 \%$ \\
\hline 7.- ¿De qué tamaño prefieres que sea el simulador? & $39 \%$ \\
\hline $\begin{array}{l}\text { 8. ¿Cuál de los siguientes simuladores estarias en condiciones de adquiriri para ti o para } \\
\text { tu escuela? }\end{array}$ & $40 \%$ \\
\hline $\begin{array}{l}\text { 9.- Crees que es importante tener simuladores clínicos en las escuelas de enfermería } \\
\text { medicina? }\end{array}$ & $78 \%$ \\
\hline $\begin{array}{l}\text { 10.- Crees que es importante practicar con un simulador antes de realizar algún } \\
\text { procedimiento con una persona? }\end{array}$ & $82 \%$ \\
\hline 11. ¿Cuánto tiempo de garantia quisiera que tuviera un simulador clínico? & $62 \%$ \\
\hline
\end{tabular}

Figura 2. Resultados generales de la pregunta 1 al 11, de la encuesta aplicada para conocer la percepción del uso de prototipos para realizar prácticas por medio de la simulación clínica.

Entre los principales resultados se encontró que El $41 \%$ de los encuestados manifiestan haber visto el procedimiento de colocación de sonda vesical, mientras que el $27 \%$ no ha visto ni ha realizado dicho procedimiento. (Fig. 3). más de la mitad de las personas refirieron que la simulación clínica, si mejora las técnicas y refuerza los conocimientos (Fig. 4). El grado de importancia d practicar antes en un simulador clínico antes que, en una persona, fue del $82 \%$, y un78\% expresó que es importante que en las escuelas existan simuladores clínicos. (Fig. 4). Y por último respecto a lo que piensan las personas de la simulación clínica cerca del $100 \%$ afirmo que es un buen método de enseñanza (Fig. 6).

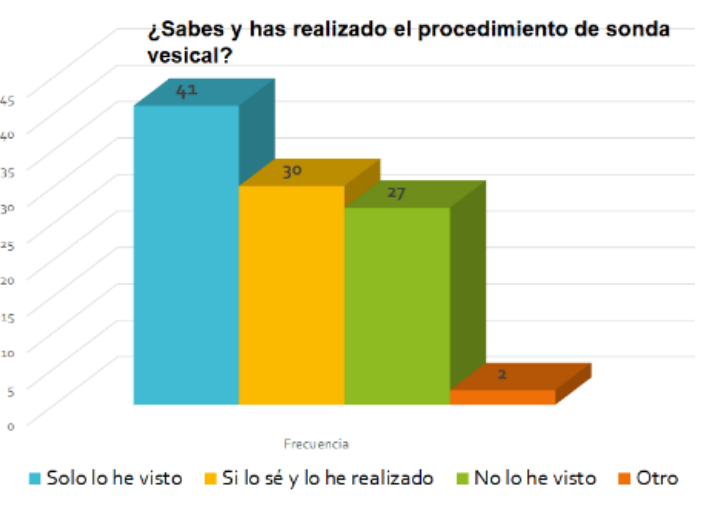

Figura 3. Resultados de la pregunta 5 de la encuesta aplicada para conocer la percepción del uso de prototipos...

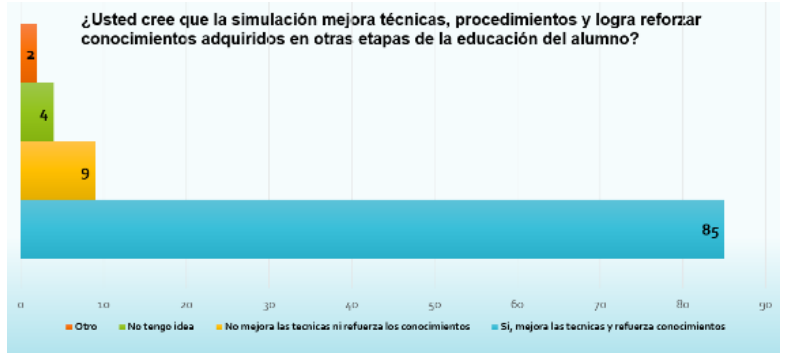


Figura 4. Resultados de la pregunta 4 de la encuesta aplicada para conocer la percepción del uso de prototipos...

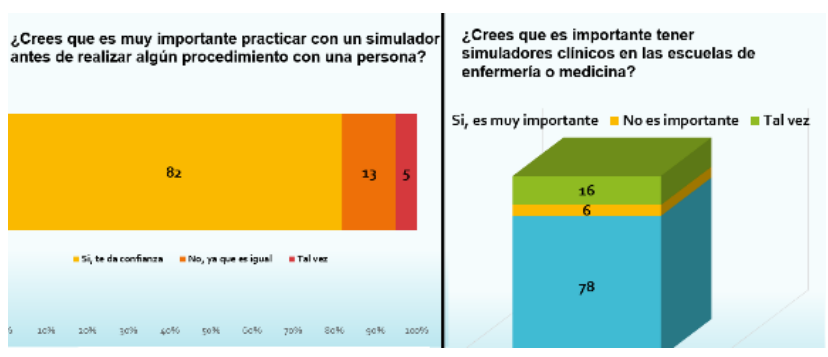

Figura 5. Resultados de las preguntas 9 y 10 de la encuesta aplicada para conocer la percepción del uso de prototipos...

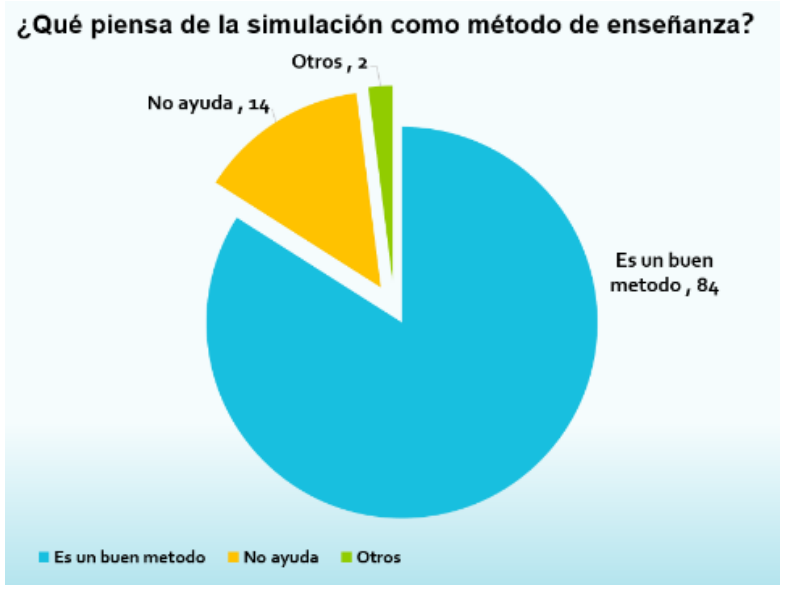

Figura 6. Resultados de la pregunta 2 de la encuesta aplicada para conocer la percepción del uso de prototipos..

Como podemos notar con estos resultados los estudiantes se expresan en positivo utilizar los simuladores clínicos como parte de sus prácticas para aprender antes de realizarlo con seres humanos.

Una razón que consideramos importante al usar el término prototipo es porque se justifica la intensión de que, al interpretar los resultados de la encuesta, resulta viable realizar en la brevedad con la experimentación del diseño para la colocación de sonda urinaria. También por la flexibilidad que otorga de ir mejorando en la elaboración de cada nueva versión, conforme a las experiencias obtenidas por el uso que hacen los estudiantes y educadores.

\section{Conclusión}

En éstos tiempo donde la práctica y experiencia, cobra mayor importancia al momento de ejercer una profesión tan delicada como lo es el área de salud. Resulta muy beneficio realizar en la etapa estudiantil; experimentos, ensayos prueba-error, que garantice la mínima o nula probabilidad de perjudicar a otros. Es importante que el docente promueva y utilice todas las herramientas necesarias ya sean modelos anatómicos, prototipos, maniquís, simuladores, entre otros; para lograr desarrollar las habilidades y destrezas que le generan confianza al estudiante de enfermería, lo que da mayor garantía al momento de realizar un buen procedimiento que cumple con el protocolo y sus respectivos indicadores., y la satisfacción que ello genera., definitivamente vale la pena estar a favor de realizar prácticas no invasivas las veces que se consideren necesarios por medio de diversos simuladores.

\section{Referencias}

[1] Dávila Cervantes A. Simulación en Educación Médica. Investigación en Educación Médica. 2014;3(10):100-5.

[2] Piña Jiménez I, Amador Aguilar R. La enseñanza de la enfermería con simuladores, consideraciones teórico-pedagógicas para perfilar un modelo didáctico. Enfermería Univ. 2015;12(3):152-9.

[3] Neri Vela, R. (2018). El origen del uso de los simuladores en medicina. Facultad de Medicina UNAM, 21-27

[4] Garcia , A., Sanchez, M., \& Merelles , A. (2012). El Practicum en el Grado de Enfermería: una propuesta de desarrollo del proyecto docente. Valencia: Recuperado de:

https://books.google.com.mx/books?id=ecpDQvE6y_YC\&pg=PA253 $\& \mathrm{dq}=$ modelos+anat $\% \mathrm{C} 3 \% \mathrm{~B} 3 \mathrm{micos}+\mathrm{did} \% \mathrm{C} 3 \% \mathrm{~A} 1 \mathrm{cticos}+\mathrm{en}+\mathrm{enfermeri}$ a\&hl=es-

419\&sa=X\&ved=0ahUKEwiJ59OsyIHoAhUIsZ4KHc3cBB8Q6AEIK

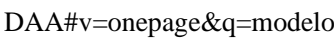

[5] Sánchez Mendiola, M., Martínez Franco, A. Informática biomédica 3ra. Edición. Facultad de Medicina de la UNAM - ELSEVIER 2018. Recuperado de:

https://books.google.com.mx/books?id=OmFbDwAAQBAJ\&pg=PA1 23\&dq=Melchor+S\%C3\%A1nchez+y+Adri\%C3\%A1n+Mart\%C3\%A Dnez,+como+la+\%E2\%80\%9Crecrear+una+situaci\%C3\%B3n+cl\%C3 $\%$ ADnica+en+un+ambiente+controlado, + de+seguridad + psicol\%C3\% B3gica,+para+el+participante, + sin+entrar+en+contacto+directo+con+ el+paciente+con+un+objeto+de+aprendizaje+o+de+evaluaci\%C3\%B3 n\&hl=es-

419\&sa=X\&ved=0ahUKEwin84zi24ToAhVIKqwKHavpD2sQ6AEIK DAA\#v $=$ onepage $\& q=$ Melchor $\% 20 \mathrm{~S} \% \mathrm{C} 3 \% \mathrm{~A} 1 \mathrm{nchez} \% 20 \mathrm{y} \% 20 \mathrm{Adri} \% \mathrm{C}$ 3\%A1n\%20Mart\%C3\%ADnez\%2C\%20como\%20la\%20\%E2\%80\%9 Crecrear\%20una\%20situaci\%C3\%B3n\%20c1\%C3\%ADnica\%20en\%2 0un\%20ambiente $\% 20$ controlado\%2C\%20de\%20seguridad\%20psicol $\% \mathrm{C} 3 \% \mathrm{~B} 3$ gica\%2C $\% 20$ para $\% 20 \mathrm{el} \% 20$ participante $\% 2 \mathrm{C} \% 20 \sin \% 20 \mathrm{ent}$ rar\%20en $\% 20$ contacto $\% 20$ directo $\% 20$ con $\% 20 \mathrm{el} \% 20$ paciente $\% 20 \mathrm{con}$ \%20un\%20objeto $\% 20$ de $\% 20$ aprendizaje $\% 20 \mathrm{o} \% 20 \mathrm{de} \% 20$ evaluaci $\% \mathrm{C}$ 3\% B3n\&f=false

[6] Lemire, Michel. (Traducción Hinke, Nina). 1993. La representación del cuerpo humano: modelos anatómicos de cera. Ciencias, núm. 32, octubre-diciembre, pp. 58-69. [En línea]. Recuperado de: https://www.revistaciencias.unam.mx/en/182-revistas/revista-ciencias32/1707-la-representaci\%C3\%B3n-del-cuerpo-humano-modelosanat $\% \mathrm{C} 3 \% \mathrm{~B} 3$ micos-de-cera.html 
Publicación semestral, Educación y Salud Boletín Científico Instituto de Ciencias de la Salud Universidad Autónoma del Estado de

Hidalgo, Vol. 8, No. 16 (2020) 212-216

[7] Real Academia Española. (2020). Obtenido de dle.rae.es/prototipo: https://dle.rae.es/prototipo

[8] Ecured. (2020). Enciclopedia de Ecuador. Obtenido de: https://www.ecured.cu/Prototipo

[9] NORMA OFICIAL MEXICANA NOM-052-SSA1-93, Que establece las especificaciones sanitarias de las sondas para drenaje urinario de hule látex natural estéril modelo Foley. Obtenido de: http://www.salud.gob.mx/unidades/cdi/nom/052ssa13.html

[10] Udepuebla.mx. Reglamento para prácticas de enfermería. Obtenido de: http://udepuebla.mx/reglamentos/reglamento-para-practicas-deenfermeria.pdf

[11] Pranatec.com (2020). Tecnología, simulación y capacitación.

Recuperado de: https://pranatec.com.mx/product/simulador-decateterismo-basic-sexo-femenino/

[12] 3B Scientific (2020). Simulador de cateterismo masculino. recuperado de: https://www.a3bs.com/thumblibrary/P93P-M/P93PM_02_1200_1200_Simulador-de-cateterismo-PRO-sexomasculino.jpg

[13] materialmedico24.es (2020) Simulador sondaje vesical. recuperado de: https://materialmedico24.es/simulador-sondaje-vesical.html 